\title{
Die Auslotung des Menschenmöglichen: Die Verschränkung von Sprache und Identität bei Hugo Loetscher
}

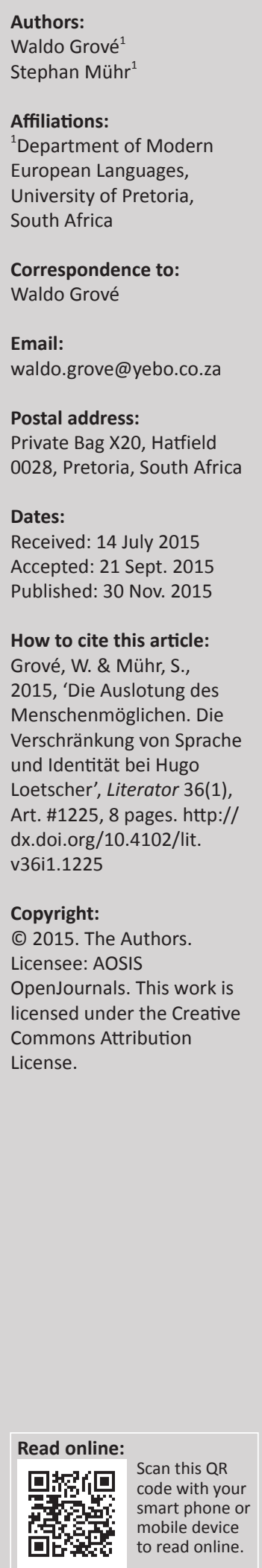

Authors:

Waldo Grove

Affiliations:

${ }^{1}$ Department of Moder

European Languages,

University of Pretoria,

Correspondence to:

Email:

Postal address:

Private Bag X20, Hatfield

14 July 2015

How to cite this article:

Menschenmöglichen. Die

Verschränkung von Sprach

Loetscher', Literator 36(1)

Art. \#1225, 8 pages. http://

dx.doi.org/10.4102/lit.

Copyright:

(c) 2015. The Authors

icensee: AOSIS

licensed under the Creative

Commons Attribution

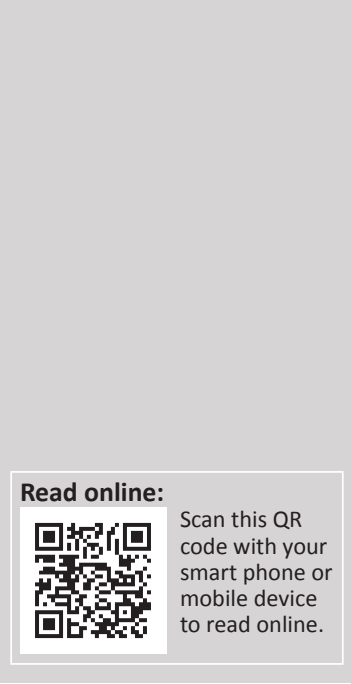

Over the course of his career as a writer, Swiss intellectual Hugo Loetscher developed a fragmentary body of ideas on the themes of language and identity respectively. In this article, it is shown that these two themes may fruitfully be compared in five aspects. Firstly, Loetscher conceives of both as being deficient and as mere potentialities. Furthermore, he desires to experience the entirety of possibilities which being human could offer, with respect to the dimensions of language and identity. These interactions with language and identity subsequently leave him with unresolved issues, of which he thinks as a remainder. Lastly, his conception of a gamut of partial identities as human possibilities is also central to a language he wishes to invent. Although Loetscher's ideas on these themes can only be extrapolated from his literary writings or essayistic fragments, a systematic treatment of these ideas in the context of philosophy of language reveals a unique and avant-garde way of thinking. This discussion leads to the realisation that Loetscher purposefully and repeatedly employs language and identity as synonymous with the possibilities of being human.

\section{Einleitung}

Der Schweizer Schriftsteller Hugo Loetscher (1929-2009) gilt als einer der 'herausragenden und führenden Vertreter' der Generation nach Frisch und Dürrenmatt (Sabalius 1995:1) und hat mit Der Immune (1985) eines der wichtigsten Romane der 'Nachkriegszeit in der Schweiz' vorgelegt (Altwegg 2005:442). Über Jahrzehnte hinweg hat sich Loetscher intensiv mit sowohl der Bedeutung von Sprache als auch von Identität (die er häufig als 'Menschenmöglichkeit' ausdrückt; siehe unten 'Das Mögliche') auseinandergesetzt, was in seinen verschiedenen Romanen, Essays und Interviews allerdings immer nur fragmentarisch erkennbar wird. Obwohl es einen umfangreichen Korpus wissenschaftlicher Arbeiten über seine literarischen Werke gibt, existiert bisher keine ausführliche Darstellung von Loetschers sprach- oder identitätsphilosophischen Reflexionen. So beschränkt sich Rosmarie Zeller in ihrem bahnbrechenden Aufsatz 'Vielsprachigkeit und Verfremdung im Werk Hugo Loetschers' (1989) weitestgehend auf die Umsetzung von Loetschers Konzept der Vielsprachigkeit in seiner literarischen Produktion; einen einzelnen Absatz (vgl. Zeller 1989:1036) widmet sie den dahinterstehenden theoretischen Überlegungen. In seinem Vergleich zwischen Loetschers Figur des Immunen und Ödipus und Grillparzers armem Spielmann bezieht sich Hinrich Seeba (2005) zwar gelegentlich auf Aussagen aus Loetschers Poetikvorlesungen, doch tut er dies im Dienste seiner eigenen vergleichenden Analyse und leistet daher keinen Beitrag zu ihrer Auslegung. Lediglich Manuel Meune (2005:79-84) setzt sich innerhalb seines Beitrags zum Thema Sprache im Werk und Leben Loetschers sehr kursorisch mit Loetschers philosophischanthropologischer Sprachauffassung auseinander. Ziel des vorliegenden Beitrags ist es daher, die Verschränkung der Themen 'Sprache' und 'Identität' in Loetschers Veröffentlichungen erstmals systematisch zu erfassen.

Loetschers Gedanken zu beiden Themen finden ihre literarische Umsetzung vor allem in seinem Hauptwerk Der Immune (1985) und dessen Folgeband Die Papiere des Immunen (1986). In Der Immune wird die (an autobiografisches Material angelehnte) Lebensgeschichte eines Individuums, das schlicht 'der Immune' genannt wird, in stilistisch äußerst heterogenen, nicht-chronologisch gereihten Kapiteln erzählt. Darüber hinaus ist der Roman extrem polyphon gestaltet, so dass seine Kohärenz in der Sekundärliteratur regelmäßig kritisiert worden ist.

Wie sehr sich Loetschers eigene Aussagen mit denen des Immunen decken, wird vor allem in seinen Poetikvorlesungen Vom Erzählen erzählen (1999) und seiner Autobiographie War meine Zeit meine Zeit (2009b) offenbar, weshalb diese Werke auch als Untersuchungsgegenstand dieses Beitrags gelten. Im Folgenden wird also sowohl auf Loetschers theoretische Schriften und Essays Bezug genommen als auch auf jene, in regelmäßigem Abstand erscheinenden 
Passagen der beiden Romane um den Immunen, die eine gewisse Konstanz aufzeigen. Loetscher bezeichnet diese als 'Zwischentexte' (1999:122ff.). Dass man die Erzählinstanz der Zwischentexte auf Loetscher beziehen darf, ist bei Cantieni (1983:179) nachzulesen. Das Umgekehrte kann man aus Sabalius (1994:374) ableiten, insofern als Loetscher einen Satz aus dem Immunen (1985:365) ohne Weiteres auf sich selbst überträgt.

Um die Verschränkung von sprach- und identitätsbezogenen Reflexionen in den genannten Werken zu erfassen, werden im Folgenden fünf aufeinander aufbauende Aspekte dargestellt, in denen beide Themen konvergieren, und zwar (1) Sprache und Identität als Defizit, (2) das Mögliche, (3) die Suche nach dem Ganzen, (4) der Rest und (5) die Wunschsprache.

Damit sollen erstmals Loetschers versprengte Ideen von der Verschränkung von Sprache und Identität systematisch zusammengefasst werden; die Darstellung ist daher zwangsläufig textimmanent. Auf sprach- und identitätstheoretische Diskurse der Entstehungszeit, also etwa auf die Auseinandersetzung mit dem Strukturalismus oder den Nachhall existenzialistischer Themen in den gesellschaftskritisch orientierten Texten der achtziger Jahre, kann diese Darstellung nicht eingehen. Denn zunächst sollen Loetschers Überlegungen selbst zu Wort kommen, ohne sie von vorn herein in linguistische oder andere Diskurse zu pressen. Ihre Relevanz liegt auch eher in ihrer eigentümlichen Vorstellung darüber, wie Sprachproduktion und Identität als 'Menschenmögliches' zusammenhängen.

\section{Der Vergleich von 'Sprache' und 'Identität' als 'Menschenmögliches' Sprache und Identität als Defizit}

Als Einführung kann man davon ausgehen, dass Loetscher ein gebrochenes Verhältnis zur Sprache (Bucher 1970:86) wie zur Identität (Loetscher 1999:131) hat. Obwohl ihm bewusst ist, dass Worte das einzige Material darstellen, mit dem er seiner schriftstellerischen Arbeit nachgehen kann (1999:30), bringt er der Sprache Misstrauen entgegen (vgl. Loetscher 1984:264). In vielen Texten (Loetscher 1984:264, 1999:96) beschreibt er es als Defizit, dass die Sprache sich nicht mit der Wirklichkeit deckt. So macht er geltend, dass die Gegenstände, die die Sprache bezeichnet, immer mehr Facetten haben, als was die Sprache aufgrund ihrer Linearität in einem gegebenen Moment zum Ausdruck bringen kann. Im gleichen Gedankengang prangert er die Polysemie der Sprache an (Bucher 1970:86). Die Beziehung zwischen Wort und Sache, zwischen Bezeichnendem und Bezeichnetem, wird also von beiden Seiten her in Frage gestellt.

Diese Unzulänglichkeit der Sprache wird im Immunen (1985) wie folgt literarisch thematisiert:

Am Ende stand ihm nichts anderes zur Verfügung als das Wort ... Aber die Worte deckten nicht ab, was sie meinten ... Was nicht aufging, war ebenso Fallgrube wie Ausgangspunkt. So sehr er darunter gelitten hatte, er hatte auch davon profitiert; er $\log$ mit dem, an das er sich klammerte. Er war ein Seiltänzer, der auf festem Grund ein Wörterbuch balancierte und der beim Jonglieren Gegenstände auffing, die er gar nicht in die Luft geworfen hatte. (S. 417)

Aus der Differenz von Wort und was es meint, zieht der Immune also seine Vorteile, seine 'Lügen', genauso wie er sich dieser Differenz ausgeliefert fühlt, weil sich daraus die Missverständnisse hinsichtlich seiner eigenen Aussagen ergeben. Die Metapher des Jongleurs von Gegenständen, die er gar nicht in die Luft geworfen hatte, veranschaulicht, wie Loetscher - als auch der Immune - der defizitären Beschaffenheit der Sprache zu entgehen sucht, nämlich indem er auf das, was sich hinter den Worten noch verbergen kann, aufmerksam macht (vgl. Loetscher 1999:96-97).

Ein konkretes Beispiel dafür ist Loetschers Erörterung der unzutreffenden 'Zweidimensionalität', die der Welt implizit zugeschrieben wird, wenn diese in Sprache gefasst wird (Loetscher 2002:80; vgl. auch Loetscher 1998b:37-43). Was versteht Loetscher hier unter Zweidimensionalität? Unabhängig vom Standort auf der Welt meint man mit dem 'Westen' immer Europa oder die Vereinigten Staaten von Amerika (USA). Beispielsweise Japan, das westlich der USA liegt, wird allerdings stets zum Osten gerechnet (z.B. Loetscher 2002:80). In der folgenden Passage aus den Papieren des Immunen (1986) wird diese Bemängelung dem Immunen in den Mund gelegt:

Und er führte sich auf, als habe er entdeckt, daß die Welt rund sei. Er ereiferte sich, links sei nicht immer links, und der Ferne Osten könne im Westen liegen, das hänge nur davon ab, von wo man aufbreche und in welche Richtung man fahre. ... Wir redeten noch immer flächig statt kugelförmig, unsere Worte täten, als müßten sie die Erdumdrehung nicht mitmachen. (S. 258)

Ein weiteres Defizit der Sprache sieht Loetscher darin, dass sie sich nicht besonders gut zur Beschreibung sinnlicher Wahrnehmungen wie etwa Geschmäcken eignet. Dies demonstriert er anhand eines frühneuzeitlichen Textes des Entdeckers Jean de Léry, der bei der Beschreibung des Geschmacks einer Ananas auf Vergleiche mit Obstsorten aus seiner heimischen Erfahrungswelt angewiesen ist (Loetscher 1999:163-164). Obwohl es de Léry nicht gelingt, seinen Lesern einen wahren Eindruck des Ananasgeschmacks zu vermitteln, gibt Loetscher (1999) zu:

Es ist denkbar, daß die dichterische Süße der Ananas so süß ist, daß die Ananas Mühe hat, mit ihrer natürlichen Süße ihr gleichzukommen. Das Defizitäre der Sprache wandelt sich also in einen Triumph, aber die dichterische Erfahrung ersetzt nicht die direkte, nämlich in die Ananas zu beißen, um zu wissen, wie sauersüß sie ist. (S. 164)

Während Loetscher sich in diesem Beispiel hinsichtlich der defizitären Beschaffenheit der Sprache versöhnlich zeigt, setzt er der entsprechenden Konzeption von Individualität 
und Identität härteste Kritik aus: Jürg Altwegg gegenüber meint er zum Beispiel, dass man nur auf dem Friedhof Identität habe, denn nur dort sei 'alles klar' (Altwegg \& Schmidt 1986:83).

Loetscher sträubt sich also gegen statische Identitätsvorstellungen, da sie zu 'Fixationen verführen' und 'verabsolutierte Identifikationen sich vorzüglich zur Konstruktion von Feindbildern eignen' (Loetscher 1999:131-132). Das Defizit, das Wort und was es meint, zur Deckung zu bringen, wird also auf das Defizit der Repräsentation eines Individuums durch einen Namen, also auf das Thema Identität, übertragen. Das kritisierte Identitätsmodell Name = Person trifft also genauso auf das kritisierte Sprachmodell Bezeichnung = Bezeichnetes $\mathrm{zu}$, wenn beide als fixiert angenommen werden. Ein Beispiel aus dem Immunen (1985) führt die Gefahren solch starrer Fixationen eindrucksvoll vor Augen:

Hätte er sich zur Deckung bringen wollen, einmal und ganz und für immer, hätte er in sich selber mindestens eine Minorität unterdrücken müssen. Damit aber wäre er sein eigener Diktator geworden und hätte in sich selber schon ein totalitäres System errichtet. (S. 366)

Gegenüber solchen Identitätszuschreibungen bevorzugt Loetscher es, sich als 'Schnittpunkt eines Beziehungsnetzes' (Loetscher 1999:131) zu betrachten. Dieses Netz ist potentiell unendlich (Sabalius 1994:374) und wird einmal von Loetscher als 'Spannungsfeld' bezeichnet (2009a). Mit 'Spannungen' sind demnach seine möglicherweise widersprüchlichen Beziehungen gemeint, aber sie gehören 'zu seiner Vorstellung von Lebendigkeit' (Loetscher 1999:131). Er ist daher 'weder darauf aus, die Spannung aufzuheben noch sie $\mathrm{zu}$ neutralisieren, sondern sie $\mathrm{zu}$ fruktifizieren.' (Loetscher 2009a)

Dass eine solch bewegliche Auffassung von Identität zweifelsohne Probleme nach sich zieht, macht folgende Stelle aus dem Immunen (1985) deutlich:

Er hatte nie ganz dazugehört. ... Und wie die meisten [seiner Gesellschaft] hatte er gemeint, er müsse sich mit etwas zur Deckung bringen, aber jede Decke, unter die er schlüpfte, erwies sich als zu klein. (S. 365)

Mit all dem, was nicht zur Deckung kam, tat sich ein Spannungsfeld auf, und sein Agieren bedeutete nicht, diese Spannungen aufzulösen, sondern sie zusammenzuhalten, darauf bedacht, nicht zerrissen zu werden. Und er war auch aus all diesen Spannungen entflohen, doch hatte er sie mitgenommen, wohin immer er auch ging .... (S. 366)

Das Wortspiel um etwas zur Deckung zu bringen, fand sich bereits im Kontext der Überlegungen zum Defizitären der Sprache. Beides bezieht sich auf den absoluten Repräsentationscharakter von Sprache beziehungsweise von Identitätszuschreibungen, den Loetscher im Falle der Identität massiv kritisiert. Das Nicht-zur-DeckungKommen, woraus das Phänomen des 'Spannungsfelds' entsteht, zeigt aber, wie komplex und letztlich literarisch Loetschers Sprachreflexionen sind; sie lassen sich nicht ohne Weiteres in eine linguistische Theorie übertragen; auch dies ist in seinem Ansatz, etwas nicht zur Deckung kommen zu lassen, enthalten.

So wird deutlich, dass Loetscher für ein 'mobiles' Modell von Identität plädiert (vgl. den englischsprachigen Titel zu Loetscher 2009a: 'Plea for a mobile identity' [Loetscher 2008]). In Anlehnung an folgendes Zitat aus den Papieren des Immunen bezeichnet Dewulf (2013a:28; vgl. auch Dewulf 2013b:129, 136) dieses Modell als 'feet-' statt 'roots-oriented':

Kaum ein Wort konnte ihn reizen, wie das Wort Wurzeln, und wenn er jemanden vom Menschen und seinen Wurzeln reden hörte, konnte er aufspringen und ihn bitten, die Schuhe auszuziehen, er möchte einmal einen sehen, der statt Füßen Wurzeln hat.

Ja, wir wollten nicht mit Wurzeln, sondern mit Füßen leben. (Loetscher 1986:172; siehe auch 1986:173, 192-194)

Diese Stelle verknüpft das Konzept der Identität zumindest metaphorisch mit dem Gehen, das dem Immunen äußerst wichtig ist: Dieser ist ein 'besessener Geher' (Loetscher 1985:123), 'auch was die Worte [betrifft]' (Loetscher 1985:417).

Zusammenfassend lässt sich bemerken, dass Loetscher die vereinseitigte, aber weit verbreitete Vorstellung, dass es zu einem Signifikanten ein Signifikat gibt, dass zu einem Individuum ein Name gehört, gerade über ihre Defizienz entlarvt. So wenig ein Name eine Identität repräsentiert, so wenig sind Signifikate eindeutige Repräsentationen von Signifikanten. Loetscher demonstriert diese Unzulänglichkeit einerseits daran, wie Eines für Vieles stehen kann (das 'Defizit' liegt dann in der misslingenden Identifizierung) und umgekehrt, wie Verschiedenes gleichzeitig mit einem Wort benannt werden kann (das 'Defizit' liegt dann in der Geltungsreichweite dieses Wortes).

\section{Das Mögliche}

Wenn jedoch die Beziehungen zwischen Name und Person und zwischen Wort und seinem Bezeichneten nicht fixiert gedacht werden können, so ergibt sich daraus zwangsläufig eine Relativierung im Modus der Aussage. Loetschers Folgerung aus der Kritik an einer statischen, abgeschlossenen Identität kommt deutlich in folgender Frage aus War meine Zeit meine Zeit (2009b:392) zum Ausdruck: 'Sind wir als Person nicht defizitär, als Individuum nur eine Möglichkeit des Menschseins lebend?' Das Stichwort des Möglichen gibt damit einen weiteren Vergleichspunkt zwischen Sprache und Identität ab, wobei Loetscher das 'Menschenmögliche' für die Knotenpunkte seines interpersonellen Beziehungsnetzes hält.

Damit geht er über die Kritik des Defizitären deutlich hinaus. Im Menschsein sind demnach viele - sogar unendliche (vgl. Sabalius 1994:374) - Möglichkeiten des Seins vorhanden, allerdings bleiben manche ungenutzt:

Was [an Möglichkeiten des Menschseins] ist nicht alles in mir ansprechbar und ungenutzt.

Auch diejenigen, auf deren Einfälle und Episoden ich selbst nie gekommen wäre, und all die, vor denen ich erschrecke, weil ihre Übeltaten in meinem Innern ein applaudierendes Echo finden. 


\section{(Loetscher 2009b:392)}

Wie unstabil und problematisch sogar die genutzten Möglichkeiten sind, macht diese Stelle aus dem Immunen (1985) deutlich:

Er gehörte den verschiedensten Majoritäten und Minoritäten an, gleichzeitig und nebeneinander, er hatte die gleiche Zugehörigkeit als Frage der Majorität und als solche der Minorität erlebt, und diese Zugehörigkeit konnte sich je nach Situation verschieben und sich im Lauf der Zeit ändern. (S. 365-366)

Als gleichermaßen nicht vorgegeben und statisch denkt und erfährt Loetscher die Sprache. Seiner Ansicht nach gibt es nämlich 'nicht Sprache, sondern nur die Möglichkeit von Sprache' (Loetscher 1999:31). Sprache ist kein stabiles Mittel, 'sondern ... ein Instrument, das im Augenblick, da es angewendet wird, stets von neuem erfunden werden muss' (Loetscher 1999:30). Damit ist nicht die neurophysiologische Konstruktionsleistung der Sprachhervorbringung gemeint; das Zitat zielt vielmehr auf eine sprachphilosophische Reflexion, dass ein Sprachsystem (etwa als Saussuresche langue) gar nicht außerhalb seiner konkreten Benutzung existiert, die sich aber bei jedem Sprechakt verändern kann. Sprache als fest vorgegebener Code oder als System einer langue wird zugunsten eines eher lebendigen, sich selbst hervorbringenden und ständig wandelbaren Organon verworfen, das eher an Überlegungen zur 'Originarität' von Sprache erinnert, wie sie etwa Leopold Peeters (2008) formuliert.

Die Problematik einer Vorstellung unendlicher Sprachpotentialität (anstelle real existierender, wenn auch immer nur in ihrer Bedeutung defizitärer Sprachen oder Worte) konnte Loetscher allerdings nicht lösen. So hofft er, die Worte, das einzige, das ihm für sein literarisches Schaffen zur Verfügung steht, 'so zusammenbringen [zu können], daß daraus Sprache entsteht' (Loetscher 1999:30).

Letzteres geschieht gar nicht zwangsläufig, denn die 'Vorstellung und Erfahrung' der Sprache als bloße Möglichkeit sollte nicht zur Annahme verleiten, man verfüge über Reichtum, da auch 'einem die Worte fehlen' können. Das beinhaltet auch 'die Verzweiflung, nicht die Worte zu finden, die man unerläßlich zum Atmen braucht’ (Loetscher 1984:264).

Diesbezüglich heißt es paradoxerweise vom Immunen, es 'fehlten ihm immer wieder die Worte. Schreiben war nichts anderes, als die Wörter zu suchen, die ihm fehlten' (Loetscher 1985:418; vgl. in Bezug auf Loetscher selbst 1999:57).

Diese 'Potentialität von Sprache' (Loetscher 1999:188) besitzt für Loetscher zwei Modi der Realisierbarkeit, einmal als ein Begriffliches, das auch als das Argumentative, das an Fakten Orientierte oder Sachliche beschrieben werden könnte, und zweitens als ein Metaphorisches (Loetscher 1999:31). Beide dieser 'Sprachen' 'hängen zutiefst zusammen und stehen im unvermeidlichen Widerspruch [zueinander]' (Loetscher 1999:31). Denn obgleich etwa eine eigentümliche, äußerst spezifische Situation im Sinne der 'Pluripotenz' von Sprache ('pluripotence' im Original: Loetscher 1990:22) nach einer von unendlichen Möglichkeiten ihrer sprachlichen Realisierung verlangen kann, ist das Begriffliche immer auch im Metaphorischen anwesend.

Und innerhalb beider dieser 'Sprachen' sind wiederum 'Sprachen' einer anderen Ordnung angelegt; Sprachen, die zu den denkbar verschiedensten und widersprüchlichsten Situationen passen, 'entsprechend der ganzen Skala, die zum Menschen und seiner Möglichkeiten gehört' (Loetscher 1999:31).

Trotz dieser durchaus verwirrenden Realisierungsmodi von Sprachpotentialitäten ist für Loetscher doch das Wichtigste, dass die jeweilige oder die jeweils realisierte (Teil-)Sprache immer situationsgerecht ist und 'Ohren hat'. (Loetscher 1999:150). Damit ist gemeint, dass sich Sprache nicht normativ von oben als Sinnsystem über eine Welt ausbreitet und damit Alleinrecht auf Sinn beansprucht, sondern umgekehrt eine (menschenmögliche) Weise ist, auf 'Welt' zu 'hören' und diese in Menschlichkeit zu transponieren. Überspitzt formuliert, verlangt Loetscher für jede Situation oder Sachlage (mindestens) eine auf sie zutreffende 'Sprache'.

In Loetschers literarischem Schaffen reihen sich daher ganz unterschiedliche Schreibstile ('Sprachen') dicht nebeneinander, um unterschiedlichen 'Erlebnislagen' zu entsprechen (vgl. Loetscher 1999:114). Im Immunen prüft er jede Sprache, die er verwendet, nur um sie zu verwerfen, indem er im nächsten Kapitel eine andere wählt (vgl. Sabalius 1994:388; Loetscher 1999:121). Damit fasst er die ‘Möglichkeiten des Erzählens als Möglichkeiten des MenschSeins' (Loetscher 1999:126) auf. Aus diesem Grund heißt es vom Immunen (Loetscher 1985):

Er ging von einer Sprache in die andere und meinte damit nicht Fremdsprachen. Von der Sprache des Wurlitzers wechselte er zur Analyse, vom Fabulieren zum Hymnus und zur Notiz und übers Lesebuch zurück zum Jargon, abrupt und dann wieder auf Übergänge bedacht. Am liebsten wäre er in alle Richtungen gegangen und aus allen Richtungen zurückgekehrt, bis jeder fremde Ort ein vertrauter wurde, jeder vertraute sich einem fremden anglich und es keinen Unterschied mehr gab zwischen vertraut und unvertraut. (S. 93; in Bezug auf Sprache vgl. auch Loetscher 1985:417)

Fazit: Im Kontext des Möglichkeitsbegriffs wird die Kritik an der (Einzel-)Sprache als System von Worten, die die Welt abdeckten, wie auch das Konzept individueller Identität (die sich aus der Summe von spezifischen Charakteristika ergäbe) hin $\mathrm{zu}$ einer unendlichen Reihe möglicher Sinnsysteme entwickelt, womit über gegebene Systeme wie langue oder Code hinausgegangen wird. Dies bringt Vorund Nachteile mit sich, die Loetscher in eindrucksvollen literarischen Bildern veranschaulicht. Als reine Möglichkeit bleibt diese Überlegung für ihn aber eine Aporie, vielleicht als Einsicht, niemals eine Sache vollständig richtig benennen zu können und umgekehrt, niemals alles auch nur erwähnt zu haben. Damit rückt der folgende Aspekt, nämlich die 
Problematik um den Absolutheitsanspruch von Identitätsund Spracherfassung in den Vordergrund.

\section{Die Suche nach dem Ganzen}

Im letzten Zitat wurde Loetschers Bedürfnis nach einer Erfahrung des ganzen Menschseins erwähnt und in einen aporetischen Zusammenhang mit der von Loetscher verspürten Defizienz von Sprache und Identität gestellt. Beides, Sprachen und Identitäten, sind menschliche Mechanismen des Kontingenzabbaus im Prozess der Welterfahrung. Durch Sprache und Identifikation wird Kontingenz in Ordnung überführt, dafür werden aber die dadurch hergestellten Relationen für fix, beziehungsweise, gültig erklärt, ohne es zu sein. Belässt man dagegen die Verhältnisse der kontingenten Welterfahrung im Vagen, Pluralen und nur Möglichen, so entbehrt der Mensch Orientierung und eine Erfahrung von Wirklichkeit oder Ganzheit.

Dieses Dilemma der Unzulänglichkeit menschlicher Epistemologie, beziehungsweise die sich erst daraus ergebende Suche nach einer Ganzheit, entfaltet Loetscher im Immunen zunächst recht konkret. In einem Brief an seine Schwester schreibt der Immune Folgendes (1985):

Ich bin nicht Vater und weiß, daß hier eine Erfahrung in meinem Leben fehlt und daß sie kaum nachzuholen ist. Ich sage das nicht, weil ich allein lebe, wenigstens hoffe ich, es nicht deswegen zu sagen, sondern es ist die Feststellung von einem, der alles erfahren will - und dann vor lauter Alles-Erfahren so vieles nicht erfährt. (S. 174-175)

Obwohl dieses Beispiel sehr spezifisch ist, kann es als pars pro toto für die allgemeine Einsicht in die immer unvollständige, unvollkommene Erfahrbarkeit der Welt als Ganzes gelten. Der Immune ist sich bewusst, dass er nie alles aus erster Hand erfahren wird. In diesem Sinne schreibt Loetscher in War meine Zeit meine Zeit (2009b):

Es sind die anderen, die Möglichkeiten von mir leben, indem sie ihre eigenen leben, und wie schön, wenn ich von jemandem hören könnte, ich hätte für ihn eine seiner Möglichkeiten gelebt. (S. 392)

Kurz darauf beschreibt Loetscher die sich daraus ergebende tiefe Abhängigkeit seines Seins von der restlichen Menschheit (Loetscher 2009b):

Wenn es darum geht, den Menschen in all seinen Möglichkeiten zu kennen, wird der andere nicht jemand, den ich toleriere, sondern jemand, den ich unerlässlich und unverzichtbar im Zeichen eines umfassenden Menschseins brauche: Ich bin erst dank seiner und aller andern ein kompletter Mensch. (S. 392) ${ }^{1}$

Die Abhängigkeit des Immunen - und Loetschers - von anderen Menschen gewinnt zusätzlich an Bedeutung, wenn man bedenkt, dass er auf der Suche nach der ihm eigenen Menschenmöglichkeit ist:

1.Auf diesen Kontext übertragen, explizieren die fast synonymen Formeln des ubuntu dieselbe Konsequenz, die sich aus dem von Loetscher hier bearbeiteten logozentrischen Dilemma von Sprache und Identität ergibt.
Zwar wollte er immer etwas werden, das es noch nicht gab, aber um zu wissen, was es noch nicht gab, war er zunächst einmal auf die Kenntnisnahme aller andern angewiesen. (Loetscher 1985:183; vgl. auch 1985:11)

Diese Kenntnisnahme seiner Mitmenschen führt zu einem problematischen Vergleich, auf den weiter unten (siehe 'Der Rest') eingegangen wird.

Eine ähnliche Sehnsucht, die Sprache in ihrer Ganzheit kennenzulernen, formulieren Loetscher und der Immune wie folgt: Beide hätten gerne zumindest einmal jedes Wort, das es gibt, benutzt (vgl. Loetscher 1999:57, 1985: 418). Mit 'geradezu schlechtem Gewissen' nimmt Loetscher daher Wörter zur Kenntnis, die er nicht kennt (Loetscher 1999:57).

Andererseits betrachtet der Immune Sprache als ein (menschenmögliches) System, das sich aus ihrer soziohistorischen Tradierung ergibt, 'weil bei jedem Wort, das er benutzte, schon immer ein anderer mitgedacht hatte' (Loetscher 1985:183). Dies steht in einem gewissen Widerspruch zur obengenannten 'Originarität' der Sprache (Peeters 2008), denn gemäß dieses Zitats entsteht durch Tradierung durchaus eine unoriginäre, gesellschaftlich normierende Sprachanschauung. Loetschers Ideen hierzu folgen aber nicht unbedingt einem linguistischen Diskurs, was am Beispiel seiner sprachlichen Erfassung von 'Zuhause' nun demonstriert werden soll.

Seine Reisen beschreibt Loetscher als Suche nach einem Zuhause; allerdings suche er nicht nach einem spezifischen Zuhause, er möchte nur sehen, was alles ein Zuhause sein kann (1998a:25). Er sucht also das Zuhause 'an sich', eine Art Summe oder Meta-Auffassung aller Möglichkeiten, die sprachlich oder schlicht konzeptionell mit 'Zuhause' ausdrückbar wären und somit ihr Ganzes bildeten.

Diese Reisen fasst er später im gleichen Gedankengang als 'Suchen nach einer runden Sprache' auf (1998a:26). Mit dem metaphorischen Adjektiv 'rund' wird einerseits auf Ganzheit und Vollkommenheit angespielt, andererseits auf die bereits erwähnte menschliche Defizienz, Sprache nur zweidimensional und nicht dreidimensional zu verstehen.

Es ist selbstverständlich, dass weder Loetscher noch der Immune - selbst wenn sie die stellvertretenden Erfahrungen Anderer in Kauf nehmen würden - je ans Ziel der 'Totalerfahrung' gelangen können. Dennoch bleibt es ein Charakteristikum von Loetschers Sprachauffassung, dass er sein Schreiben als Versuch erklärt, die 'Totalität der Möglichkeiten [menschlicher Rollen]' in der 'Totalität der Sprachen' (vgl. Sabalius 1994:388) einzufangen.

\section{Der Rest}

Wenn aber die Ganzheit von Aussage- oder Menschenmöglichkeiten realiter nicht umgesetzt oder ausgesprochen werden kann, entstehen daraus zwangsläufig 
Fragen nach den Resten in den unvollständigen oder defizitären Versprachlichungen von Welterfahrung und Identität.

Wie oben angedeutet, versuchen Loetscher und der Immune über einen Vergleich mit Anderen herauszufinden, wer sie sind. In dem Maße, in dem der Immune sich allerdings nicht vollkommen mit anderen Individuen deckt, entsteht ein 'Rest' (Loetscher 1985; vgl. u.a. 1985:60, 44):

Wo immer er sich zur Deckung bringen wollte, es ging nie ganz auf. Er begriff, es blieb immer ein Rest, stets von neuem ein Rest und immer wieder ein anderer, und diese gesammelten Reste machten ihn aus. (S. 365)

Ein ähnlicher Rest entsteht beim Einsatz der Sprache: 'Nicht nur mit dem, was ihn ausmachte, blieb stets ein Rest, sondern auch mit jedem Wort' (1985:418).

Allerdings hofft der Immune, innerhalb dieses Rests (der ihn ausmacht), unter den 'noch nicht genutzten Möglichkeiten', jene zu finden, die 'auf seine eigne Weise etwas Menschen-Mögliches zu sein [erlauben]' (1985:366). Dies veranschaulicht, wie schmal der Grat zur Individuation nach Loetschers Vorstellung ist.

Das Gleiche kann aus folgender Passage im Immunen gefolgert werden:

Als wichtigstes Vorgehen erwies sich immer wieder der Versuch, von der eigenen Person abzusehen und sie als Anlaß zu nehmen, um von anderem zu erfahren. Dabei blieb stets ein Rest. Er wußte oft nicht, wohin damit, und konnte ihn nur wieder bei sich unterbringen. Dieser Rest war mit seinen Gegebenheiten erfaßbar; er lieferte Daten, mit denen man einen Computer fütterte. Sein Lieblings-Ausweis neben dem $\mathrm{Paß}$ war daher die Computer-Karte: eine bestimmte Anzahl von Löchern in einer bestimmten Anordnung auf einem Stück Papier; wäre eines der Löcher nur um einen Millimeter daneben gestanzt, hätte die Karte bereits einem andern gehört. So nahm er zuweilen ein solches Papier vor die Augen als Guckkasten und sah durch die Löcher, die ihn ausmachten. (Loetscher 1985:60)

In dieser Analogie wird die Problematik der Ganzheit oder vollkommenen Bestimmung von Identität in gewisser Weise 'auf den Punkt gebracht'; es ist daher kein Zufall, dass Loetscher (1999) selbst auf dieses literarische Bild in Vom Erzählen erzählen unter die Rubrik 'Sprache' verweist. Die Beziehung dieser Stelle zur Vorstellung von einer Ganzheit von Sprache als Menschsein wird deutlich, wenn man sie zusammen mit folgender Passage liest, die ebenfalls aus dem Band Vom Erzählen erzählen stammt:

Erst durch die andern und dank ihnen erlangen wir Bewußtsein von uns selbst. Indem wir all die andern Sprachen und deren Möglichkeiten kennen, finden wir zurück zur eigenen und zu deren Möglichkeiten - selbst auf das Risiko hin, daß am Ende meine Sprache nur aus ein paar Worten besteht, aber es wären die unabdingbar eigenen. (Loetscher 1999:32)

Beide Passagen entwickeln die Schwierigkeit, Individualität nicht in Opposition zum Kollektiv, sondern durch es zu erfassen, andererseits aber nicht in ihm aufzugehen. Dabei steht die politische Dimension weniger im Vordergrund, sondern wirklich die Frage nach dem Verhältnis von Sprache und Identität. Es ist davon auszugehen, dass Loetscher, der alle Worte, die es gibt, zumindest einmal in den Mund nehmen möchte, sich nicht damit zufriedengeben würde, nur seine ureigenen Vokabeln zu benutzen, wenn er sie einmal ermittelt hat. Viel wahrscheinlicher würde er seiner eigenen Forderung nachgehen und heraushören, genau nach welcher 'Sprache' eine jeweilige Situation verlangt, ohne jedoch gänzlich auf seine Eigenheit zu verzichten.

\section{Die Wunschsprache}

Die Darlegung der bisherigen sprach- und identitätstheoretischen Aspekte von der Defizienz zur Vermöglichung, zur Ganzheitsfrage und zum Rest folgte einer mehr oder weniger logischen Kette. Dabei zeichnete sich ab der Vermöglichung allerdings die Aporie zwischen empirisch gegebenen oder gesellschaftlich normierten Fixationen (mit all ihren Defiziten) und dem gewünschten, aber nicht realisierbaren Absolutheitsanspruch sprachlicher Identität ab. Um zu zeigen, wie diese beiden Auffassungen von Sprache und Identität in Loetschers Konzept des Menschenmöglichen konvergieren, soll abschließend die Beschreibung einer 'Wunschsprache' des Immunen (Loetscher 1985) erörtert werden, weil darin eine Art Versöhnung zwischen empirischer Defizienz und erwünschter Ganzheit anklingt:

[Er] machte sich auch daran, eine Sprache zu erfinden, für die er sammelte, was er in toten und lebenden Sprachen als Errungenschaften auffaßte, in Grammatik, Syntax und Wortschatz. Zum Beispiel war es eine Sprache: wo bei 'wir' unterschieden wird, ob es zwei oder mehr sind, wo auf jeden Superlativ ein Konjunktiv folgt und wo 'ich' mehr als einen Nominativ hat. Eine Sprache, die er nicht als Vehikel verstand, sondern die immer genauere und feinere Unterscheidungen traf und mit ihren Regeln auf so viele menschliche Möglichkeiten wie nur denkbar Rücksicht nahm. (S. 418) ${ }^{2}$

Die ethische Vorstellung, dass diese Sprache dazu dienen soll, möglichst viele Menschenmöglichkeiten zu berücksichtigen, anstatt lediglich Kommunikationsmittel zu sein, ist ein durchaus eigentümlicher Gedanke Loetschers. Damit ist das Menschenmögliche abermals mit der Sprache verknüpft und umgekehrt. Die Sprachauffassung des Immunen (und Loetschers) neigt damit entschlossen zum Anthropologischen und Nicht-Mechanistischen.

Loetscher selbst berichtet, wie er Wörterbücher sammelt nicht mit dem Ziel des Sprachenlernens, sondern lediglich 'um einen Einblick zu gewinnen in das, was sonst noch an Sprache möglich ist' (Loetscher 2009b:226).

2.Es ist zu vermuten, dass mit dem "ich", das mehr als einen Nominativ hat, auf das

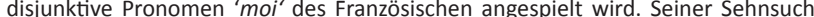
nach einem Dualis im Deutschen widmet Loetscher einen längeren Absatz in Wor meine Zeit meine Zeit (Loetscher 2009b:227-228): "Im Deutschen gibt es für war" meine Zeit meine Zeit (Loetscher 2009b:227-228): "Im Deutschen gibt es für "ich" nur ein einziges Wort und nur eines für "wir". Ich habe stets bedauert, dass wir nich wie im Griechischen oder im Russischen einen Dual haben, ein eigenes "wir" fü die private und kleinere Gemeinschaft. Ich bin für die sprachliche Kollektivierung des "ich". Was, wenn eine Sprache zehn "wir" unterschiede, weil das familiäre „wir nicht das gleiche ist wie das der Arbeitsgruppe, das „wir" der Intimität nicht das des Massenaufmarsches, das der Verschwörung nicht das der Solidarität - wie wohl das globalisierte „wir" lautet?" 
Das Projekt, eine neue Meta-Sprache aus allen bestehenden Sprachen zu extrapolieren, passt auch zur bereits diskutierten Suche nach der Ganzheit. Insofern als man annehmen kann, dass eine solche 'Supersprache' von Natur aus 'rund' sein würde und 'Ohren hätte', kann man daraus auch die Zuversicht herauslesen, dass die Sprache als Medium letztendlich doch zu rehabilitieren ist.

Die 'Idiosynkrasie' (Loetscher 1985:417) des Immunen, eine - logisch unmögliche - Steigerung von 'anders' als 'anderser' zu formulieren, bezeugt Loetschers Auffassung, dass es ein 'Recht auf Unterschiede und auf Anderssein gibt und daß aus diesem Verschieden- und Anderser-Sein keine Wertung abgeleitet werden' dürfe (Loetscher 1985:418). Diese Idee stellt eine anschauliche Versprachlichung von Loetschers Identitätsauffassung und seinem akuten Interdependenzbewusstsein dar.

Die bisherigen Ausführungen haben dargelegt, wie sehr sich 'Sprache' und 'Menschenmöglichkeit' im Denken Hugo Loetschers trotz gewisser Unterschiede ähneln. Die folgende vollkommene Gleichsetzung beider Konzepte mag daher wenig verwundern:

Aber ob die Entdeckungen [auf meinen Reisen] mich zum Experten oder zum Analphabeten machten, was mich dabei immer wieder in Erstaunen versetzte, war die Erfahrung, wie viele Sprachen der Mensch spricht - und dies nicht linguistisch verstanden. Ein Staunen darüber, was dem Menschen alles zum Thema Mensch einfiel. (Loetscher 1999:26)

Nach Wolfgang Frühwald (1999) beschreibt Loetscher:

$[D]$ ie vielen Sprachen der Menschheit ... ja nicht nach den Regeln von Grammatik und Stilistik, sondern im Sinne einer abenteuerlichen Entdeckungsreise in den Kosmos, vielleicht sogar in das Chaos des Humanen. (S. 44)

\section{Fazit}

In diesem Aufsatz wurde die engste Verknüpfung von der Sprach- und der Identitätsauffassung Hugo Loetschers anhand einer argumentativen Reihe von fünf Aspekten nachgewiesen, wie sie sich in seinen Publikationen findet und sich in Loetschers Begriff des Menschenmöglichen am fasslichsten manifestiert. So konnte gezeigt werden, dass es sich bei Loetschers Gleichsetzung von Sprache und Menschsein, die im Kontext der Reflektionen über seine Reiseerfahrungen wie urplötzlich erscheint, keineswegs um einen isolierten oder spontanen Einfall handelt. Vielmehr stellt diese Gleichung den Höhepunkt einer subtilen und doch intensiven Beschäftigung mit Sprache und Identität dar, die verfolgbare Spuren in Loetschers Werk hinterlassen hat.

Die hier herangezogenen Primärtexte sind allesamt literarischer oder essayistischer Natur; ein eigener sprach- und identitätsphilosophischer Ansatz lässt sich aus diesen Quellen durch die hohe Interdependenz der hier nachgewiesenen fünf Aspekte zwar annehmen, nicht aber notwendigerweise beweisen, was hinsichtlich des Motivs des Nicht-zur-Deckung-Bringens wahrscheinlich systemimmanent, beziehungsweise intendiert ist. Vor diesem Hintergrund bleibt es ein Desideratum, eines der ganz wenigen wissenschaftlichen Arbeiten von Loetscher selbst, nämlich seine schwer zugängliche Dissertation 'Der Philosoph vor der Politik. Ein Beitrag zur Politischen Philosophie' (1956) auf das Konzept des Menschenmöglichen zu untersuchen.

\section{Anerkennungen Konkurrierende Interessen}

Die Autoren erklären, dass sie keine finanzielle oder persönliche Beziehungenhaben, die sie beim Schreiben dieses Artikels unangemessen beeinflusst haben könnten.

\section{Autorenbeiträge}

This article emanated from discussions regarding the MA dissertation on 'Die Immunitätsmetapher als kohäsionsstiftendes Element in Hugo Loetschers "Der Immune"' by W.G. (University of Pretoria) (supervisor: S.M. [University of Pretoria]), submitted and accepted cum laude in 2015. For this article, W.G. wrote the line of argument with regards to the primary sources. S.M. advised on the theoretical and methodological approach, editing and proofreading the manuscript accordingly.

\section{Bibliographie}

Altwegg, J., 2005, 'Ein Existentialist - aber mit Witz' in J. Dewulf \& R. Zeller (Hrsg.) In alle Richtungen gehen: Reden und Aufsätze über Hugo Loetscher, S. 439-444, Diogenes, Zürich.

Altwegg, J. \& Schmidt, A., 1986, 'Hugo Loetscher. Die Schweiz ist kein Sonderfall', in J. Altwegg \& A. Schmidt (Hrsg.), Perspektive Schweiz: Gespräche mit Zeitgenossen, S. 63-84, Benziger, Zürich.

Bucher, W., 1970, 'Hugo Loetscher: "Für eine Literatur der Behaftbarkeit", in W. Bucher \& G. Ammann (Hrsg.), Schweizer Schriftsteller im Gespräch: Bd. I, S. 73-108, Reinhardt, Basel.

Cantieni, B., 1983, 'Hugo Loetscher', in B. Cantieni (Hrsg.), Schweizer Schriftsteller persönlich: Interviews, S. 173-189, Huber, Frauenfeld.

Dewulf, J., 2013a, 'Reimagining Heimat from a hybrid perspective: Hugo Loetschers concept of a plural Heimat', in G. Eichmanns \& Y. Franke (eds.), Heimat goes mobile: Hybrid forms of home in literature and film, pp. 14-36, Cambridge Scholars Publisher, Cambridge.

Dewulf, J., 2013b, 'Vom Diskurs in der Enge zum Diskurs in die Weite: Hugo Loetschers Konzept der "Pluralen Heimat" als Schlüsselbegriff in der neueren Literatur der deutschsprachigen Schweiz', The German Quarterly 86(2), 123-140. http://dx.doi. org/10.1111/gequ.10174

Frühwald, W., 1999, 'Am Anfang aller Entdeckungen', in H. Loetscher (Hg.), Vom Erzählen erzählen: Poetikvorlesungen, S. 35-46, Diogenes, Zürich.

Loetscher, H., 1956, 'Der Philosoph vor der Politik. Ein Beitrag zur politischen Philosophie', Dissertation, Universität Zürich.

Loetscher, H., 1984, 'Minerva und die Musen', in G. Sütterlin (Hg.), Das Hugo Loetscher Lesebuch, S. 264, Diogenes, Zürich.

Loetscher, H., 1985, Der Immune: Roman, 2. Aufl., Diogenes, Zürich.

Loetscher, H., 1986, Die Papiere des Immunen: Roman, Diogenes, Zürich.

Loetscher, H., 1990, 'La création littéraire et la pluripotence de la langue', in D. Barthélemy (Hg.), Les écrivains face à la critique: Les actes du Ve Colloque interdisciplinaire, Fribourg, S. 19-29, Editions universitaires, Fribourg.

Loetscher, H., 1998a, 'Reisender unter Reisenden', Quarto. Zeitschrift des Schweizerischen Literaturarchivs 9(10), 13-26.

Loetscher, H., 1998b, Der Waschküchenschlüssel, oder, Was - wenn Gott Schweizer wäre, Diogenes, Zürich.

Loetscher, H., 1999, Vom Erzählen erzählen: Poetikvorlesungen, erw. Neuausg., Diogenes, Zürich.

Loetscher, H., 2002, 'Die runde Sprache des Reisens', Der Deutschunterricht 54(4), 78-80.

Loetscher, H., 2008, 'Plea for a mobile identity', Transit 4(1), angesehen am 7. Jul 2015, bei https://escholarship.org/uc/item/8jd063wq. 
Loetscher, H., 2009a, 'Schweizstunde', Die Zeit 22. April 2009, eingesehen am 4. Juli 2015, bei http://www.zeit.de/2009/17/CH-Loetscher.

Loetscher, H., 2009b, War meine Zeit meine Zeit, Diogenes, Zürich.

Meune, M., 2005, 'Von Sprache(n) in den Welten Hugo Loetschers - eine Perspektive aus dem "anderen Lateinamerika"', in J. Dewulf \& R. Zeller (Hrsg.), In alle Richtungen gehen: Reden und Aufsätze über Hugo Loetscher, S. 77-101, Diogenes, Zürich.

Peeters, L., 2008, 'Taalvermoë en originariteit', Acta Academica 40(2), 28-52.
Sabalius, R., 1994, 'Ein Erstaunen darüber, was es an Welt gibt: Ein Interview mit Hugo Loetscher', Dimension: Contemporary German Arts and Letters 20, 368-397.

Sabalius, R., 1995, Die Romane Hugo Loetschers im Spannungsfeld von Fremde und Vertrautheit, Lang, New York.

Seeba, H.C., 2005, 'Das Lachen des helvetischen Ödipus', in J. Dewulf \& R. Zelle (Hrsg.), In alle Richtungen gehen: Reden und Aufsätze über Hugo Loetscher, S. 183-213, Diogenes, Zürich.

Zeller, R., 1989, 'Vielsprachigkeit und Verfremdung im Werk Hugo Loetschers', Schweizer Monatshefte. Zeitschrift für Politik, Wirtschaft, Kultur 69(12), 1035-1043. 